\title{
REFLEXÕES SOBRE PROMOÇÃO DA SAÚDE DO IDOSO NAS CONFERÊNCIAS NACIONAIS DE SAÚDE
}

ANA SANTOS'; GUIMARÃES, IRACI"

\section{RESUMO}

Promoção da Saúde é a viabilidade de meios para população de melhorar sua saúde e obter maior controle sobre a mesma. Entende-se por envelhecimento um processo natural do ciclo da vida, singular, contextual, marcado por perdas e descobertas de novas estratégias, que pode ser analisado por diferentes focos: psicológicos, sociológicos, biológicos entre outros. Conferências de Saúde são caracterizadas como instâncias institucionalizadas, colegiadas, de discussões e deliberações sobre questões de saúde. O objetivo deste estudo foi analisar limitações e avanços do conceito de promoção da saúde do idoso nas Conferências Nacionais de Saúde; a metodologia foi qualitativa, com leituras reflexivas, centrando-se a análise nos termos: idoso e terceira idade. Nos relatórios das Conferências $10^{\circ}, 11^{\circ}, 12^{\circ}$ e $13^{\circ}$, houve avanço na abordagem da promoção da saúde do idoso, mas, ainda, essa temática merece atenção com mais debates sobre o processo de envelhecimento, comprometimentos disso no âmbito público e conseqüências para a Saúde em todos os seus aspectos.

Palavras - chave: idoso, envelhecimento, promoção da saúde, conferência de saúde

\section{INTRODUÇÃO}

Conforme literatura sobre envelhecimento, não há um atendimento adequado para população idosa no Brasil ${ }^{[18 ; 24]}$, desta forma é apresentado ao cenário de políticas públicas a imediata necessidade de buscar meios para alcance da longevidade com ampliação da qualidade de vida. O desafio que se coloca para este século é o de como cuidar de uma população com mais de 32 milhões de idosos, sendo o perfil desses, em

I. Assistente Social - UnB. Pós-graduanda em Educação em Promoção da Saúde -UnB:

II. Psicóloga - UnB. Mestre em Ciências da Saúde - UnB.; 
sua maioria, cidadãos com baixos níveis sócio-econômico e educacional. ${ }^{[24]}$ Nesse contexto, as ações de saúde pública merecem destaque, sobretudo porque a população idosa apresenta alta propensão a doenças, especialmente, crônicas, transtornos cerebrais e mentais. ${ }^{[18 ; 24]}$ Diante disto, o objetivo do trabalho foi analisar limitações e avanços do conceito de promoção da saúde do idoso explicitados nos relatórios da $10^{\mathrm{a}}, 11^{\mathrm{a}}, 12^{\mathrm{a}}$ e $13^{\mathrm{a}}$ Conferências Nacionais de Saúde. ${ }^{[6 ; 7 ; 8 ; 9]}$

\section{ENVELHECIMENTO}

A velhice é um processo natural do ser humano, ou qualquer outro ser, sendo analisada por diversos parâmetros como psicológicos, sociológicos, jurídicos e biológicos. Os mais difundidos podem ser categorizados como: "biológico/ comportamental", "economicista", "socioculturalista" e "transdisciplinar". [26]

Na perspectiva transdisciplinar, mais ampla, a velhice é um "... fenômeno natural e social que se desenrola sobre o ser humano, único, indivisível, que na sua totalidade existencial, defronta-se com problemas e limitações de ordem biológica, econômica e sociocultural que singularizam seu processo de envelhecimento." [26, p. 8] Já, como um fenômeno "biológico/comportamentalista" as características da degeneração natural do organismo são mais evidenciadas. O destaque é o idoso como portador de diversas patologias, as quais devem ser retardadas num esforço conjunto entre sociedade e indivíduo. ${ }^{[26]}$

Outros esforços são concentrados em delimitar o envelhecimento como um fenômeno "economicista". O foco passa a ser a retirada do indivíduo do mundo produtivo, especialmente, na questão da aposentadoria. Os idosos são aqui reconhecidos como agentes de direitos e como tal devem lutar por aquilo que é assegurado por lei, prevê a possibilidade de uma nova forma de ação social e não uma interrupção da vida em sociedade. Ganha destaque questões previdenciárias, como valor da aposentadoria e má utilização do recurso público. ${ }^{[26]}$

Outro segmento menciona a velhice como um fenômeno "sócioculturalista", o qual reconhece como insuficiente os olhares das perspectivas "biológico/comportamentalista" e "economicista". Tem como pressuposto que a sociedade/cultura determina as funções e competências preferenciais para cada idade, seja no seio da família ou na divisão social do trabalho. ${ }^{[26]}$

Alguns conceitos sobre envelhecimento convergem quando mencionam perdas, bem como, quando retratam a descoberta de estratégias para vivenciar este momento. 
Por fim, ser idoso é aquele indivíduo com 60 anos ou mais, saudável, ou seja, que não tenha suprimida a capacidade de decidir e executar seus próprios desígnios, portanto, com a autonomia e a independência preservadas. ${ }^{[2 ; 18 ; 24 ; 26]}$

Portanto envelhecimento saudável, em uma perspectiva de saúde ampliada, pode ser compreendido como uma conquista pelo resultado positivo da interação de diferentes fatores: físicos, mentais, independência na vida diária, integração social, apoio familiar e independência financeira. A saúde para os idosos não quer dizer ausência de problemas, estes serão comuns nas diferentes fases da vida, mas sim o equilíbrio entre os vários aspectos da capacidade funcional destes cidadãos. ${ }^{[24 ; 29]}$

\section{PROMOÇÃO DA SAÚDE}

A moderna concepção de saúde pressupõe estratégias para além da ausência de doenças e volta-se para o bem-estar e qualidade de vida. ${ }^{[14 ; 15 ; 16]} \mathrm{O}$ conceito ampliado de saúde a reconhece como o produto resultante das condições de alimentação, habitação, renda, educação, meio ambiente, trabalho, emprego, transporte, lazer, liberdade, acesso e posse de terra e acesso a serviços de saúde. ${ }^{[5]}$

Na Carta de Ottawa ${ }^{[13]}$, promoção de saúde engloba o fornecimento de estratégias para a população, que visem melhorar sua saúde, bem como proporcionar um maior controle sobre a mesma, como: fortalecer a ação comunitária, construir políticas públicas saudáveis, criar ambientes de suporte, desenvolver habilidades pessoais, e reorientar os serviços de saúde.

Goodstall et al (2001, apud $\left.{ }^{[1]}\right)$ indicaram alguns dos elementos que são apontados pela promoção da saúde: 1) metas que incluem a saúde e a qualidade de vida, não apenas na prevenção da morbidade; 2) maior atenção na igualdade, justiça e participação, além de, conceito holístico da saúde; 3) empowerment, ou seja, atribuição de poder ao individuo; 4) análise macrossocial; e, 5) enfatizar os princípios da Carta de Ottawa.

Assis et al ${ }^{[1]}$ apresentaram uma avaliação exploratória do Projeto de Promoção da Saúde do Núcleo de Atenção ao Idoso, da Universidade Aberta da Terceira Idade, na Universidade do Estado do Rio de Janeiro, e concluíram que esse Projeto foi uma experiência benéfica para os idosos, promovendo suas capacidades de lidar positivamente com o envelhecer, gerando saúde. Os objetivos alcançados, em curto prazo, foram: disseminação de informações, reforço da autoestima, e favorecimento dos 
contatos sociais. Sobre o autocuidado e bem-estar subjetivo, a pesquisa indicou uma estabilização positiva; o Projeto foi apontado como um instrumento que potencializou a reorientação das práticas de saúde, favorecendo a atenção integral, humanização dos serviços, participação dos sujeitos acerca de questões relacionadas à saúde e bem-estar, sejam elas na esfera individual ou coletiva. ${ }^{[1]}$

Em outra pesquisa, com o título de "Grupo Feliz Idade: cuidado de enfermagem para promoção da saúde na terceira idade" ${ }^{28]}$ mostrou-se a experiência de formação de um grupo desenvolvido por enfermeiras do Programa Saúde de Família, na capital do Ceará, o qual contou com a colaboração de outros profissionais: médico, educador físico e fisioterapeuta, e seus resultados apontaram que as atividades iniciais do grupo, que eram atividades físicas, recreativas e oficinas, foram ampliadas com caminhadas, alongamentos, recreação, atividades comemorativas, oficinas para discussão de assuntos da comunidade, passeios e terapia comunitária. Logo, conforme a pesquisa, o trabalho deste grupo apresentou alterações significativas nos aspectos bio-psicossociais dos envolvidos, utilizando-se de participação de todos, incentivando a autonomia dos idosos na realização das ações propostas. ${ }^{[28]}$

Assim, esse trabalho referencial, mostrou que, como reflexo diferenciado, a formação do grupo influenciou na rotina de outra instituição, a qual despertou para necessidade de atenção a população idosa, disponibilizando horários específicos para hidroginástica e atendimento odontológico, serviços anteriormente não oferecidos pela instituição a esta população. ${ }^{[28]}$

Em outro contexto, Martins et al ${ }^{[21]}$ descreveram uma experiência com educação em saúde para cuidadores de pessoas idosas, em domicílio; e, o objetivo foi identificar e classificar as necessidades de educação em saúde referidas por cuidadores de idosos. Foram apontadas como principais necessidades educativas aspectos relacionados às doenças e seus agravos, a questão medicamentosa, exercícios físicos e alimentação. Retrataram o despreparo técnico por parte dos cuidadores informais investigados, porém, registraram o potencial de investimento em educação em saúde nesta área, fazendo referência ao interesse dos cuidadores pelo aprendizado e os conhecimentos dos mesmos advindos da prática cotidiana. Como estratégia a educação em saúde, mencionam como primeira etapa a interatividade entre educador, idoso e cuidador, num segundo momento, sugerem colocar o conteúdo em prática e por fim, fazer do cuidado um hábito diário. Martins et al ${ }^{[21]}$ informaram que favorecer a prática de projetos viáveis 
tendo em vista as condições pessoais e do meio ambiente em que vivem o idoso pode ser uma alternativa interessante.

Descrevemos o papel da promoção da saúde do idoso, mas cabe registrar que a velhice é um processo que vem se construindo ao longo de todas as fases da vida, por este fato ressalta-se a importância de mencionarmos que a promoção da saúde não é importante somente neste momento. Apesar de o contexto mundial estar requerendo ações imediatas para o público idoso, por conta do envelhecimento global, as ações de promoção da saúde devem estar presentes em todas as fases da vida.

Nesse sentido, a pesquisa "Experiência extramural em hospital público e a promoção da saúde bucal coletiva" [20] teve o objetivo de registrar uma experiência de promoção da saúde da boca, baseada na integração ensino-serviço, em um hospital, com intuito de compreensão dos vários determinantes do processo saúde-doença. Propôs-se uma avaliação de desempenho dos alunos de odontologia, enquanto educadores em saúde bucal, com foco na integralidade e atendimento humanizado. O resultado mostrou o caráter fundamental de implantar atividades educativas e preventivas em saúde bucal, em ambiente hospitalar. Quanto ao paciente/acompanhante, essa estratégia foi uma oportunidade para reconhecê-los de forma mais integral, promovendo um melhor entendimento entre saúde bucal e saúde geral. Além disso, menciona o efeito multiplicador das informações trabalhadas para gerar promoção da saúde no núcleo familiar. ${ }^{[20]}$

Partindo do pressuposto que a promoção da saúde tem como objetivo criar meios para a população gerar e controlar sua saúde, adotando estratégias, como fortalecimento e participação do usuário; diante do fenômeno envelhecimento populacional de modo acelerado, alterando perfil demográfico e epidemiológico; e conhecendo as peculiaridades desta fase da vida: velhice, refletir sobre o debate de como a promoção da saúde do idoso está sendo compreendida em fóruns de participação da sociedade, torna-se uma possibilidade de reconhecendo as ausências de fala instigar na prática profissional a colaboração para o preenchimento das lacunas no debate.

\section{CONSELHOS DE SAÚDE E CONFERÊNCIAS NACIONAIS DE SAÚDE}

Os Conselhos de Direito surgiram como respostas às reivindicações de movimentos sociais e ganharam destaque com a promulgação da Constituição Federal de 
1988, em vários setores político-sociais do Brasil, inclusive na saúde. Os conselhos caracterizam-se como um espaço público, composto por governo e sociedade, institucionalizados, legalmente constituídos, tendo como função basilar a consolidação do exercício da democracia e o controle social. Podem ser definidos como um espaço de discussão, de formação de opinião pública e tomador de decisões, as quais devem ser consideradas pelo Poder Público. ${ }^{[17 ; 22]}$

Os Conselhos de Saúde acolhem as demandas da sociedade, inclusive, deliberando sobre o plano de saúde local, e, aprovando a proposta orçamentária para o setor, sendo as demandas consubstanciadas pelas Conferências de Saúde. ${ }^{[2]}$

Desde 1990, as Conferências de Saúde são definidas como instâncias colegiadas, institucionalizadas, de controle social. Representam, juntamente, com os conselhos de saúde, a efetivação do controle social (entendido aqui como controle das ações estatais pela sociedade) junto ao Sistema Único de Saúde - SUS. A Conferência tem como objetivo a avaliação da situação de saúde e proposição de diretrizes para a formulação da política de saúde nos níveis correspondentes, acontecendo de quatro em quatro anos, com representantes dos diferentes segmentos sociais. ${ }^{[10 ; 2]}$

\section{METODOLOGIA}

O estudo objetivou analisar os marcadores históricos das conferências de saúde, com metodologia qualitativa, em leituras reflexivas de revisões e resumos das publicações, construindo questionamentos sobre as limitações e avanços do conceito de promoção da saúde do idoso explicitados nos relatórios de $10^{\mathrm{a}}, 11^{\mathrm{a}}, 12^{\mathrm{a}}$ e $13^{\mathrm{a}}$ Conferências Nacionais de Saúde. Esses relatórios são mais setoriais, e as demandas apresentadas nasceram em debates regionais.

Conforme a resolução 196/96 do Conselho Nacional de Saúde ${ }^{[4]}$ e pelos preceitos do Comitê de Ética da Faculdade de Ciências da Saúde, não foi necessário aprovação por não se tratar de trabalho científico que envolva o ser humano direta ou indiretamente.

\section{RESULTADOS}

\section{1)Relatório da $10^{\mathrm{a}}$ Conferência Nacional de Saúde}

$\mathrm{Na} 10^{\mathrm{a}}$ Conferência Nacional de Saúde apesar de apresentar em seu texto "atenção integral à saúde da Terceira Idade", não alcançou a complexidade do tema: promoção da saúde do idoso. Incluiu em suas propostas a melhoria da saúde, mas não 
ficou clara a intenção de ir além da prevenção da morbidade, apesar de registrar termos como "promoção da vida e da saúde" em atividades coletivas.

Não houve nos trechos analisados estimativas de participações dos idosos enquanto ativos na promoção da saúde; sem mérito para a justiça social, equidade ou empowerment como eixos privilegiados de ação para esta faixa etária. Esse relatório nem mesmo registrou a necessidade de uma política pública voltada para esse público, tratando o assunto como "ações de atenção integral à saúde". Porém, houve previsão de atividades coletivas, entretanto, sem contemplar o desenvolvimento de habilidades pessoais, ações comunitárias, entre outros aspectos da promoção da saúde.

Nessa Conferência ocorreram discussões sobre saúde do idoso, o quê pode ter sido o início de tentativas de responder a uma demanda real, o envelhecimento da população. Algumas demandas do processo de envelhecimento foram compreendidas, como por exemplo, a adoção do termo Terceira Idade evitando discriminações inerentes a outras denominações que se destinam a esta faixa etária. Contudo, caracterizou-se como um processo que homogeneíza o idoso, não privilegiando a idiossincrasia da fase como reflexo das histórias de vida de cada um, suas experiências pessoais.

O relatório não abordou questões como, por exemplo, o momento de retirada o indivíduo do mundo produtivo. Assim, pode-se inferir uma predominância na centralidade das práticas de hábitos saudáveis, quando ocorre somente mudança de hábitos individuais que resultam na promoção da saúde, o quê parece insuficiente e ultrapassada, sendo necessários, deste modo, diferentes olhares para garantir uma leitura menos incompleta.

\section{2)Relatório da $11^{a}$ Conferência Nacional de Saúde}

$\mathrm{Na} 11^{\mathrm{a}}$ Conferência Nacional ocorreu um debate sobre a ampliação e qualificação das ações voltadas à saúde do idoso, na qual houve menção a elementos-chave de promoção da saúde como: divulgação de direitos assegurados a esta população, e, o diálogo entre as diversas ações do Estado destinadas a atender aos idosos, ampliando na prática o conceito de saúde. Houve destaque para espaços de controle social, com uma leitura macro da saúde do idoso, das políticas sociais integradas, sugerindo, assim, uma reavaliação das políticas sociais para os idosos.

Apesar do avanço na discussão sobre promoção da saúde do idoso em relação à $10^{a}$ Conferência, permaneceram os desafios de enfrentar o debate ampliado do processo de envelhecimento, pois não surgiram propostas que atrelaram previdência e saúde do idoso, trabalho e empregabilidade na terceira idade, transporte adequado às 
necessidades desta fase da vida, processo de desenvolvimento urbano e situação da saúde do idoso, esportes e lazer propícios a esta idade entre outros temas pertinentes à velhice.

\section{3)Relatório da $12^{\mathrm{a}}$ Conferência Nacional de Saúde}

$\mathrm{Na} 12^{\mathrm{a}}$ Conferência apresentaram-se valores como justiça social e participação, com possibilidade de garantir o fortalecimento do controle social e a sugestão de disseminação de informações sobre os direitos e benefícios sociais dos idosos.

Ocorreu um breve debate sobre articulação entre políticas, com preocupação em garantir o cumprimento da Política Nacional do Idoso, de 1999, e do Estatuto do Idoso, de 2003, indicando um rompimento com a "lógica do favor" - paternalista - uma vez que reconheceu o idoso enquanto portador de direitos. Por fim, aqui, solicitou-se a criação de condições reais para cumprimento das referidas legislações.

Ainda, nessa Conferência, mencionou-se a situação dos cuidadores de idosos e a necessidade de cadastrar e fiscalizar as instituições de longa permanência, com o foco no usuário do serviço, pois estas ações referentes às instituições asilares deveriam centrarse na integração social do idoso e nas condições dignas e humanizadas de atendimento.

Esse relatório atendeu a muitos dos pressupostos da promoção da saúde, mais assim como o anterior não se aprofundou em questões complexas do processo de envelhecimento.

\section{4)Relatório da $13^{a}$ Conferência Nacional de Saúde}

$\mathrm{Na} 13^{\mathrm{a}}$ Conferência propuseram-se ações pertinentes a outras áreas de Governo, como a redução da idade para obtenção do Benefício de Prestação Continuada - BPC (benefício para pessoa com mais de 64 anos, que não podem prover sua subsistência e da família), previsto na Lei Orgânica da Assistência Social - LOAS, de 1993, também, previu o enfrentamento da discriminação, solicitou a criação de mecanismos para operacionalizar o direito à saúde, respeitando o Estatuto do Idoso e prevendo a divulgação de seu conteúdo e mencionou a necessidade da disseminação de informações mais claras sobre medicamentos.

Quanto ao acesso ao BPC, este pode ser entendido como uma medida de proteção social, tendo relação estreita com desigualdade social, que é um dos eixos de trabalho prioritário na Promoção à Saúde. No relatório há, também, uma preocupação com a implantação, em todas as esferas da Federação, de Programas de Atenção Domiciliar e ampliação dos centros de convivência e de terapia familiar para os familiares de idosos. 
A $13^{a}$ Conferência pautou a necessidade de elaboração de planos de atenção à saúde do idoso com recorte de gênero, raça, classe, geração, orientação sexual e condições físicas (respeitando o Estatuto do Idoso e a Política Nacional do Idoso), modificando a aparente idéia de que todo idoso tem as mesmas necessidades. Além disso, solicitou o aceleramento de implantação de Redes Estaduais de Assistência à Saúde do Idoso (estabelecida pela Política Nacional do Idoso) e vislumbrou a necessidade de ações de estímulo do Governo Federal para a criação de Secretarias de Atendimentos aos Idosos.

Foi registrada a questão do financiamento relacionado especificamente à saúde do idoso em dois momentos: o compartilhado entre as três esferas do governo (Federal, Estadual e Municipal) para implantação de centros de convivência e centros de permanência para pessoas sem família; e, o das três esferas de Governo, na garantia de implementação, ampliação e permanência dos serviços de referência para população idosa.

Nessa Conferência, o idoso foi apresentado de forma mais integral, até mesmo no que tange a implementação de um conselho que o trate como um todo, e não somente os aspectos separados de sua vida; ela, também, o destacou como aquele que se constrói dentro de um contexto social (prevê ações que levam em consideração as diferenças dentro desta faixa etária).

\section{CONSIDERAÇÕES FINAIS}

As leituras reflexivas sobre os relatórios da $10^{\mathrm{a}}, 11^{\mathrm{a}}, 12^{\mathrm{a}}$ e $13^{\mathrm{a}}$ Conferência Nacional de Saúde mostraram que houve avanços na abordagem da Promoção da Saúde dos idosos, mas merece atenção o debate ampliado da compreensão do que seja o processo de envelhecimento, para quê, ao fim, assim como o conceito de saúde, possamos construir um conceito ampliado da velhice na prática do sistema de saúde.

Algumas observações pertinentes a todos os relatórios merecem destaque:

1) Pouco debate sobre financiamentos específicos para ações em saúde do idoso - a Promoção da Saúde está diretamente relacionada à implementação de políticas saudáveis, assim são necessários, também, relevância nos aspectos financeiros; uma vez que, programar ações e não desencadear recursos necessários para sua execução é apenas criar uma falsa sensação de conquista, que pode ser entendida como uma expressão da correlação de 
forças na arena de construção das políticas públicas, ou seja, onde a prioridade não foi à efetivação do direito; ${ }^{[25]}$

2) Falta de ênfase no apoio à pesquisas sobre envelhecimento - o conhecimento científico é um subsídio para "o cuidar" do idoso, compreendendo necessidades humanas básicas e as possíveis adaptações que ocorrem ao longo da vida. Principalmente em pesquisas que recorrem a aspectos biológicos, psicológicos, sociais, culturais e espirituais, favorecendo o distanciamento do olhar somente sobre a patologia e centrando-se nos indivíduos envolvidos;

3) E, a pouca atenção à necessidade de capacitação profissional para atuação junto ao público analisado - na perspectiva interprofissional, é necessário a formação de profissionais treinados especificamente para atender esta faixa etária; os profissionais de saúde têm papel chave na criação de estratégias para fortalecer a discussão em favor da promoção da saúde do idoso

Sugere-se o fortalecimento de estratégias que visem incluir no debate da sociedade a identificação de causas tratáveis de déficit cognitivo e de perda de independência, com o objetivo de se manter a capacidade funcional do idoso, com independência e autonomia, e, que, por sua vez, focalizem em sua qualidade de vida como eixo central da promoção da saúde. A questão do envelhecimento se tornou uma demanda às políticas públicas, pois ações de promoção, prevenção e recuperação na área da saúde da pessoa idosa não foram pensadas, de modo adequado ou suficiente, para a chegada deste novo século e suas peculiaridades. Parece que o Brasil não se preparou para esta realidade: um contingente significativo de novos idosos e idosos que estão envelhecendo cada vez mais.

O enfrentamento da discriminação também pode ajudar a construir o caminho para uma sociedade mais equânime, e a disponibilização de informações se feita de modo adequado pode contribuir para o empowerment. A educação em saúde, pode, também, viabilizar meios de um cuidado mais seguro, para fortalecimento tanto do idoso, quanto dos cuidadores, e, de Programas e Ações de cuidados em domicílio, que pode ser uma alternativa interessante no processo de promoção da saúde. De tal modo, os centros de convivência, casas-lar, serviços domiciliares podem surgir como alternativas para superar opções existentes, como por exemplo, instituições asilares que não priorizam a promoção da saúde do idoso. ${ }^{[23]}$ Ressalta-se que o estabelecimento de redes de contatos sociais 
também é um mecanismo para promover um envelhecimento ativo, pois esta alternativa pode auxiliar a minimizar o isolamento social a que muitos idosos estão sujeitos. ${ }^{[27]}$

Por fim, refletir sobre as demandas trazidas pelas Conferências Nacionais de Saúde traz à tona a necessidade de amadurecimento da sociedade sobre o processo de envelhecer e suas conseqüências individuais e sociais.

\section{REFERÊNCIAS BIBLIOGRÁFICAS:}

1. Assis, Mônica de et al. Avaliação do projeto de promoção da saúde do Núcleo de Atenção ao Idoso: um estudo exploratório. Interface (Botucatu) vol. $13 \mathrm{n}^{\circ} 29$ Botucatu abr/jun 2009. Disponível em http://www.scielo.br/scielo.php? pid=S1414-32832009000200010\&script=sci abstract\&tlng=pt, acessado em janeiro de 2010.

2. Brasil, Lei $\mathbf{n}^{\circ} \mathbf{1 0 . 7 4 1}$, de $1^{\circ}$ de Outubro de 2003. Dispõe sobre o Estatuto do Idoso e dá outras providências. Disponível em http://www.planalto.gov.br/ccivil/LEIS/2003/L10.741.htm, acessado em janeiro de 2010.

3. Brasil, Lei $\mathbf{n}^{\circ} \mathbf{8 . 7 4 2}$, de 7 de Dezembro de 1993. Dispõe sobre a organização da Assistência Social e dá outras providências. Disponível em http://www.planalto.gov.br/ccivil_03/LEIS/L8742.htm, acessado em janeiro de 2010.

4. Brasil. Conselhos Nacional de Saúde. Resolução $n^{\circ} 196$, de 10 de outubro de 1996. Aprova as seguintes diretrizes e normas regulamentadoras de pesquisas envolvendo seres humanos. Disponível em http://conselho.saude.gov.br/resolucoes/1996/Reso196.doc, acessado em janeiro de 2010.

5. Brasil. Ministério da Saúde. Conselho Nacional de Saúde. Relatório Final da $\mathbf{8}^{\mathbf{a}}$ Conferência Nacional de Saúde. Brasília, 1986. Disponível em http://conselho.saude.gov.br/biblioteca/Relatorios/relatorio_8.pdf, acessado em janeiro de 2010.

6. Brasil. Ministério da Saúde. Conselho Nacional de Saúde. Relatório Final da $\mathbf{1 0}^{\mathbf{a}}$ Conferência Nacional de Saúde. Brasília, 1992. Disponível em http://conselho.saude.gov.br/biblioteca/Relatorios/relatorio_10.pdf, acessado em janeiro de 2010.

7. Brasil. Ministério da Saúde. Conselho Nacional de Saúde. Relatório Final da $\mathbf{1 1}^{\mathrm{a}}$ Conferência Nacional de Saúde. Brasília, 2000. Disponível em http://conselho.saude.gov.br/biblioteca/Relatorios/relatorio_11.pdf, acessado em janeiro de 2010.

8. Brasil. Ministério da Saúde. Conselho Nacional de Saúde. Relatório Final da $\mathbf{1 2}^{\mathbf{a}}$ Conferência Nacional de Saúde. Brasília, 2003. Disponível em http://conselho.saude.gov.br/biblioteca/Relatorios/relatorio 12.pdf, acessado em janeiro de 2010.

9. Brasil. Ministério da Saúde. Conselho Nacional de Saúde. Relatório Final da $\mathbf{1 3}^{\mathbf{a}}$ Conferência Nacional de Saúde. Brasília, 2008. Disponível em http://conselho.saude.gov.br/biblioteca/Relatorios/relatorio_13.pdf, acessado em janeiro de 2010 . 
10. Brasil. Ministério da Saúde. Lei 8.142, de 28 de Dezembro de 1990. Dispõe sobre a participação da comunidade na gestão do Sistema Único de Saúde (SUS) e sobre as transferências intergovernamentais de recursos financeiros na área da saúde e dá outras providências. Disponível em http://www.planalto.gov.br/ccivil_03/LEIS/L8142.htm, acessado em janeiro de 2010.

11. Brasil. Ministério da Saúde. Portaria n 2.528, de 19 de outubro de 2006. Aprova a Política Nacional de Saúde da Pessoa Idosa. Disponível em http://portal.saude.gov.br/portal/arquivos/pdf/2528\%20aprova\%20a \%20politica\%20nacional\%20de\%20saude\%20da\%20pessoa\%20idosa.pdf Política Nacional do Idoso (2006), acessado em fevereiro de 2010.

12. Brasil. Ministério da Saúde. Resolução Conselho Nacional de Saúde № 333, de 04 de novembro de 2003. Aprova diretrizes para criação, reformulação, estruturação e funcionamento dos conselhos de saúde. p.5 Disponível em http://elegis.anvisa.gov.br/leisref/public/showAct.php?id=8604\&word=\#', acessado em Janeiro de 2010.

13. Brasil. Ministério da Saúde. Secretaria de Políticas de Saúde. Projeto Promoção da Saúde: as cartas de promoção da saúde. Brasília, 2001

14. Buss, Paulo Marchiori. Promoção da saúde e qualidade de vida. Ciênc. saúde coletiva vol.5 $\mathrm{n}^{\circ} .1$ Rio de Janeiro 2000, disponível em http://www.scielosp.org/scielo.php?pid=S141381232000000100014\&script=sci arttext acessado em julho de 2009.

15. Buss, Paulo Marchiori. Promoção e educação em saúde no âmbito da Escola de Governo em Saúde da Escola Nacional de Saúde Pública. Cad. Saúde Públic vol.15 suppl.2 Rio de Janeiro 1999, disponível em http://www.scielosp.org/scielo.php?pid=S0102311X1999000600018\&script=sci arttext acessado em janeiro de 2010.

16. Campos, Gastão Wagner et al. Avaliação de política nacional de promoção da saúde. Ciênc. Saúde coletiva vol 9 n $^{\circ} 3$ Rio de Janeiro Julho/Setem. 2004. Disponível em http://www.scielo.br/scielo.php?pid=S141381232004000300025\&script=sci arttext, acessado em outubro de 2009.

17. Correia, Maria Valéria Costa. Controle Social na Saúde. in Mota, Ana Elizabete [et al] (orgs). Serviço Social e Saúde. São Paulo: OPAS, OMS, Ministério da Saúde, 2006.

18. Guimarães, Iraci Gonçalves. Quando esquecer é o problema: Representações Sociais de familiares sobre saúde mental no envelhecimento e os desafios impostos pela demência. Dissertação (Mestrado) - UnB, Faculdade de Ciências da Saúde, Brasília, 2005.

19. Jardim, Viviane Cristina Fonseca da Silva et al. Um olhar sobre o processo de envelhecimento: a percepção de idosos sobre a velhice. Rev Brás. Geriatr. Gerontol. v. $9 \mathrm{n}^{\circ} 2$ Rio de Janeiro, 2006. Disponível em http://www.unati.uerj.br/tse/scielo.php?script=sci_arttext\&pid=S180998232006000200003\&Ing=pt\&nrm=iso, acessado em novembro de 2009.

20. Junior, Antonio Medeiros et al Experiência extramural em hospital público e a promoção da saúde bucal coletiva. Rev. Saúde Pública 2005; 39: 305-10. Disponível em http://www.scielo.br/scielo.php?pid=S003489102005000200024\&script=sci_arttext, acessado em março de 2010.

21. Martins, Josiane de Jesus et al. Necessidades de educação em saúde dos cuidadores de pessoas idosas no domicílio. Texto \& Contexto - enferm. v. $16 \mathrm{n}^{\circ}$ 
2 Florianópolis abr/jun. 2007. Disponível em http://www.scielo.br/scielo.php? pid=S0104-07072007000200007\&script=sci_abstract\&tIng=pt, acessado em novembro de 2009.

22. Oliveira, Mariana Siqueira de Carvalho. Fragmentos de discursos construídos a várias vozes: notas sobre democracia, participação social e Conselhos de Saúde in Costa, Alexandre Bernardino et al (org.). Direito achado na rua: introdução crítica ao direito à saúde. Brasília: CEAD/UnB, 2008.

23. Pestana, Luana Cardoso \& Santo, Fátima Helena do. As engrenagens da saúde na terceira idade: um estudo com idosos asilados. Rev esc.enferm. USP v.42 n² São Paulo jun.2008. Disponível em http://www.scielo.br/scielo.php? pid $=$ S0080-62342008000200009\&script=sci_abstract\&tIng=pt, acessado em janeiro de 2010.

24. Ramos, Luiz Roberto. Fatores determinantes do envelhecimento saudável em idosos residentes em centros urbanos: Projeto Epidoso, São Paulo. Cad. Saúde Pública vol. $19 \mathrm{n}^{\circ} 3$ Rio de Janeiro jan.2003. Disponível em http://www.scielo.br/scielo.php?pid=S0102311X2003000300011\&script=sci_arttext, acessado em janeiro de 2010.

25. Sampaio, Juliana et al. Análise das políticas públicas: uma proposta metodológica para estudo no campo da prevenção da Aids. Rev. Brás. Saúde Matern. Infant., Recife, 6 (3): 335-346, jul./set. 2006. Disponível em http://www.scielo.br/scielo.php?script=sci_arttext\&pid=S1519-

38292006000300010 , acessado em outubro de 2009.

26. Siqueira, Renata Lopes de et al. A velhice: algumas considerações teóricas e conceituais. Cienc. Saúde Coletiva vol. 7 n 4 Rio de Janeiro, 2002. Disponível em $\quad$ http://www.scielo.br/scielo.php?script=sci_arttext\&pid=S141381232002000400021 , acessado em novembro de 2009. p.8

27. Souza, Luccas Melo \& Lautert, Liana. Trabalho voluntário: uma alternativa para promoção da saúde do idoso. Rev. Esc. Enferm. USP v. $42 n^{\circ} 2$ São Paulo jun. 2008. Disponível em http://www.scielo.br/scielo.php? script=sci_arttext\&pid=S0080-62342008000200022, acessado em Janeiro de 2010.

28. Victor, Janaína Fonseca et al. Grupo Feliz Idade: cuidado de enfermagem para a promoção da saúde na terceira idade. Rev. Esc.de Enferm. USP v. 41 São Paulo Dezembro de 2007. Disponível http://www.scielo.br/scielo. php?pid=S008062342007000400026\&script=sci_abstract\&tlng=pt, acessado em Janeiro de 2010.

29. World Health Organization. Envelhecimento ativo: uma política de saúde. Tradução Suzana Gontijo. Brasília: Organização Pan-Americana de Saúde, 2005. 